\title{
High Frequency Sound Based Device Communication
}

\author{
Sahil Lakhwani ${ }^{1}$, Nishant Pardamwar ${ }^{2}$, Nikhil Khewalkar ${ }^{3}$ \\ Department of Computer, S.S.B.T. C.O.E.T, Jalgaon, India ${ }^{1}$ \\ Department of Computer, S.S.B.T. C.O.E.T, Jalgaon, India ${ }^{2}$ \\ Department of Computer, S.S.B.T. C.O.E.T, Jalgaon, India ${ }^{3}$
}

\begin{abstract}
In the chronicle of wireless communication of devices, there have been various attempts at providing a seamless short distance communication technology. All of those technologies are hardware specific. Adding them to consumer devices influences various factors like cost and software compatibility. This paper shows use of ultrasonic sound as data carrier over short range between two modern devices, capable of generating and detecting ultrasound only through their standard speakers and microphones. The results show that such communication is possible over a distance of few metres in a frequency range of $20 \mathrm{KHz}$ to $21 \mathrm{KHz}$
\end{abstract}

Keywords: wireless device communication; short range technologies; ultrasound; Goertzel;

\section{INTRODUCTION}

Modern computing and communication devices offer a wide range of wireless communication protocols to transmit data. The popular technologies embedded in consumer devices are Bluetooth[1], and IEEE802[2]. All these communication systems need their own hardware components. They also suffer from other issues. RF systems can suffer from security issues. Infrared can suffer from light interference.

Again, since data transferring between devices does not require any extra equipment and is implemented on the software level, this technology can be used to exchange info between phones with different operating systems, e.g. iOS, Android, BlackBerry, Windows Phone, etc. This is particularly relevant today, during a time of increasing mobile platform differentiation and growing demand for cross-platform communication.

Another approach is to use ultrasound for communication in air. The main advantage of the ultrasound approach is that it works with almost every modern consumer device, for example smart phones. It only requires a speaker, a microphone, and a relatively modern processor, powerful enough to perform all necessary calculations. High frequency sound communication uses audible sounds as a low bandwidth data channel and can enhance, for example, smart phone usability. Moreover, it doesn't change the fundamental devices people use to communicate. Instead, it can exploit the existing programmable interfaces of modern smart phones to augment modes of communication that people already know and use. The scope of this technology over other short range communication and authentication technologies can be summarised as

\section{- $\quad$ Alternative to NFC.}

NFC technology has been around for several years now but still hasn't received wide recognition nor did it get into large-volume production. Mobile industry leaders still can't agree on a single universal format for the protocol of near field communication. NFC technology, on the other hand, requires a special chip to be included in the hardware. different OS

\section{Gesture recognition}

The ultrasound technology could be applied not only for data transferring but for gesture recognition as well. For Android there are already application prototypes that recognize user's gestures through a camera. The same idea could be realized using ultrasound.

\section{RELATED WORK}

Although high frequency sound technology has been old and extensively used in various fields, there have been less attempts to use it for low bandwidth data communication.

An Israeli start-up SlickLogin[3] has attempted a solution to make the two-factor system by allowing users to complete the two-step process simply by placing the phone next to their laptop or tablet. In another attempt a company named Azoft[4] uses different techniques to make the ultrasound communication more efficient. The R\&D team at Azoft has tried so by using different techniques such as Fast Fourier Transformation, and heterodyne based demodulator. A wireless keyboard using ultrasound has been designed [5].

A study presented in [6] has been undertaken of ultrasonic communications methods in air, using a quadrature modulation method. Simulations were performed to establish the likely performance of quadrature phase shift keying over the limited bandwidth available in an ultrasonic system. Quadrature phase shift keying 
modulation is then implemented within an experimental Algorithm: Goertzel communication system.

\section{ULTRASONIC COMMUNICATION}

The human ear can hear sounds in the range of approximately $20 \mathrm{~Hz}$ to $20 \mathrm{KHz}$. Any frequencies above and below this range are imperceptible to the ear. Standard audio equipment including speakers, microphones and amplifiers is designed to work in this frequency range. Mobile devices can theoretically record and reproduce sounds with a peak frequency as high as $22050 \mathrm{~Hz}$. This limit is caused by the maximum possible output sample rate of those devices, which is equal to $44100 \mathrm{~Hz}$. So the frequencies higher than $20 \mathrm{KHz}$ and lower than $22050 \mathrm{KHz}$ can be used. So, we have a frequency range in which devices can transmit and receive inaudible sounds or the range for the carrier frequency. Now we need to choose a modulation technique, a scheme that defines how the carrier signal is controlled by a modulating signal. The choice of modulation technique affects the implementation complexity of the modulator and demodulator and errorrate performance at a given speed. Various demodulating techniques can be used, such as demodulator based on Fast Fourier Transform (FFT). FFT outputs the full frequency spectrum of the signal, which is good, but it is to be made sure that the number of FFT bins matches the number of harmonics, i.e. to decompose the signal into 1024 harmonics we need 1024 FFT bins. It turned out that we couldn't analyse the signal in our frequency range at satisfactory transmission rate though. Goertzel algorithm produces the same results, but with a performance increase, since it analyses selectable harmonics and not the whole spectrum. Hence the Goertzel algorithm can perform tone detection using much less CPU horsepower than the Fast Fourier Transform.

\section{A. Goertzel Algorithm}

Goertzel algorithm does not output the entire spectrum.

The algorithm is used here to detect if a particular frequency is present in the recorded buffer. Instead of generating interrupt for each sample, each buffer of sample is processed. So Goertzel is a much faster method if only a few number of frequencies are to be detected.

The basic Goertzel [7] gives the real and imaginary frequency components as a regular Discrete Fourier Transform (DFT) or Fast Fourier Transform (FFT) would. Magnitude and phase can then be computed from the realimaginary pair.

The requirements for the algorithm are as follows:

Require: N (Goertzel block size), target freq (frequency to be detected) sample rate (the pre-selected sampling rate), $Q_{0}, Q_{1}, Q_{2}\left(Q_{1}\right.$ is just the value of $Q_{0}$ last time. $\mathrm{Q}_{2}$ is just the value of $\mathrm{Q} 0$ two times ago)
1: Compute the constants:

$2: \mathrm{k}=($ int $) 0.5+\frac{\mathrm{N} * \text { (target_freq ) }}{\text { (sample_rat) }}$

$3: \mathrm{w}=(2 * \mathrm{n} / \mathrm{N}) * \mathrm{k}$

4: $\operatorname{cosine}=\cos \mathrm{W}$

5: $\operatorname{sine}=\sin \mathrm{w}$

6: coeff $=2 *$ cosine

7: for all sample blocks do

8: $\quad Q_{1}=0$

9: $\mathrm{Q}_{2}=0$

10: for all samples do

11: for all $i$ such that $i \mathrm{~N}$ do

12: $\quad \mathrm{Q}_{0}=$ coeff $* \mathrm{Q}_{1}-\mathrm{Q}_{2}+$ sample

13: $\quad \mathrm{Q}_{2}=\mathrm{Q}_{1}$

14: $\quad \mathrm{Q}_{1}=\mathrm{Q}_{0}$

15: $\quad$ end for

16: $\quad$ end for

17: real $=\left(\mathrm{Q}_{1}-\mathrm{Q}_{2} *\right.$ cosine $)$

18: $\operatorname{imag}=\left(\mathrm{Q}_{2} *\right.$ sine $)$

19: magnitude $^{2}=$ real $^{2}+$ imag $^{2}$

20: end for

A simple threshold test of the magnitude decides if the frequency was present or not.

Goertzel block size $\mathrm{N}$ is like the number of points in an equivalent FFT. It controls the bin width. For example, the sampling rate is $8 \mathrm{KHz}$ and $\mathrm{N}$ is 100 samples, then the bin width is $80 \mathrm{~Hz}$. This would steer towards making $\mathrm{N}$ as high as possible so as to get the highest frequency resolution. But the higher $\mathrm{N}$ gets, the longer it takes to detect each tone, simply because you have to wait longer for all the samples to come in. For example, at $8 \mathrm{kHz}$ sampling, it will take $100 \mathrm{~ms}$ for 800 samples to be accumulated. Another factor influencing choice of $\mathrm{N}$ is the relationship between the sampling rate and the target frequencies. The frequencies are to be centered in their respective bins. In other words, target frequencies should be integer multiples of sample rate/N. N doesn't

have to be a power of two.

A debouncing technique is used which requires multiple detections in a row before reporting a tone's presence.

Examples of the values calculated according to the algorithm are shown below. Two threshold and deboune values are used which are chosen according to the physical surroundings.

$\stackrel{-}{N}=420$

Example 1: For target frequency $21000 \mathrm{KHz}$

debounceValue $=13$;

debounceValue2 $=5$;

THRESHOLD = 1300;

THRESHOLD2 = 8000;

sampling rate $=44100$;

target frequency $=21000$;

coeff $=-1.977662$

sine $=-0.988831$;

cosine $=0.05219642$ 
- $\quad$ Example 2: For target frequency $20700 \mathrm{KHz}$

$\mathrm{N}=450$

debounceValue $=13$;

debounceValue2 $=5$;

THRESHOLD = 1300;

THRESHOLD2 = 8000;

sampling rate $=44100$;

target frequency $=20700$;

coeff $=-1.961910310698383$;

sine $=0.19423435121997193$;

cosine $=-0.9809551553491915$

\section{B. Protocol Design}

For the system developed, the protocol used is a simple handshake mechanism. Frequencies $21000 \mathrm{KHz}$ and $20700 \mathrm{KHz}$ are used for transmitting ' 1 ' and ' 0 ' respectively. An acknowledgement from receiver is a ' 1 ', transmitted to sender. To detect a lost bit, sender waits for a fixed time before assuming that a bit was not received and then resending that bit. Similarly, receiver has its own timeout value after which it assumes that there is no more bit to be received, and hence terminates the communication.
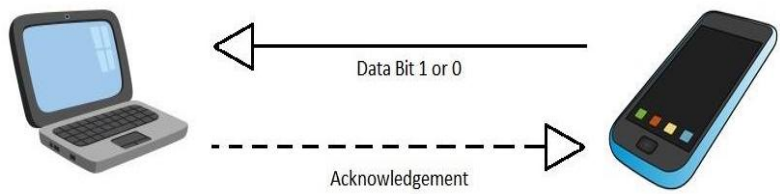

Figure 1(a) Mobile to laptop

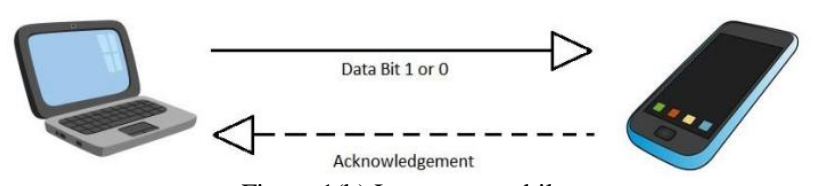

Figure 1(b) Laptop to mobile

\section{ANALYSIS}

The actual physical communication is tested under normal room conditions with a Nexus phone and a Sony Vaio laptop. The chosen target frequencies are $21000 \mathrm{KHz}$ and $20700 \mathrm{KHz}$ with threshold values 1300 and 8000 .

The magnitude values computed for target frequency $21000 \mathrm{KHz}$ is shown as an example, illustrating its presence or absence is shown below.

For frequency $21000 \mathrm{KHz}$, when not found

MAGNITUDE 527.8632722273217

MAGNITUDE 229.77114321214987

MAGNITUDE 715.217500255635

MAGNITUDE 692.1885286909423

MAGNITUDE 943.9969541606276

MAGNITUDE 43.6138159474666

MAGNITUDE 280.43276754044126

MAGNITUDE 91.97743244953975

MAGNITUDE 211.04319850258807

Copyright to IJARCCE
MAGNITUDE 349.32525549723965

MAGNITUDE 1150.973601882521

MAGNITUDE 565.5315458732673

MAGNITUDE 568.274467826947

MAGNITUDE 450.07908258709585

MAGNITUDE 428.49078674065385

MAGNITUDE 496.24775533225875

MAGNITUDE 791.7256899159931

MAGNITUDE 199.4868699343878

MAGNITUDE 250.90418619251997

MAGNITUDE 889.5424393582229

MAGNITUDE 325.4611925266914

For frequency $2100 \mathrm{KHz}$, when found

MAGNITUDE 18248.524191994366

MAGNITUDE 19044.975491090834

MAGNITUDE 8799.01567363818

MAGNITUDE 10672.750388360402

MAGNITUDE 7783.1133147247465

MAGNITUDE 4979.461437236895

MAGNITUDE 4500.5070748358485

MAGNITUDE 1305.6026624944766

MAGNITUDE 1341.4903979293051

MAGNITUDE 2693.884897608045

MAGNITUDE 1400.8516331874266

MAGNITUDE 7463.0131314931805

MAGNITUDE 8644.622898107258

The above values of magnitude for show that magnitude values greater than threshold value were successively received. After the such high values were received more times than the debounceValue2, the frequency is confirmed for it presence

\section{RESULTS AND DISCUSSIONS}

The readings shown in above section show that communication is seamless. Repeatedly, bits were sent from sides, i.e. mobile and laptop. Packets of 5 bits, 8 bits and 12 bits were tested for accuracy and efficiency.

Each packet was sent 10 times from either side. A 5 bit was correctly received 9 times. 8 bit packet was correctly transferred 8 times. For the 12 bit packet accuracy was 6 out of 10 times. Thus, the overall accuracy can be calculated as about 76 percent.

The results are taken in normal room conditions. Surrounding environment can have a serious effect, leading to interference. This decreases the accuracy. As per the results, the error in a packet is not more than 2 bits. Moreover, there is no error correction method implemented. A standard error correction can easily detect and correct two bits.

\section{CONCLUSIONS}

High frequency sound communication can be used for device-to-device communication. The proposed system can be easily accommodated in any standard device 
without any hardware overhead. This system can be very reliable and easy to use. This type of communication is claimed to be replacing some popularly used short range technologies. In further workings, the challenge is to iterate the testing for pure communication by implementing various modulating and demodulating techniques, error checking methods and encryption techniques.

\section{REFERENCES}

[1] J. P. K. Gilb, "Bluetooth Radio Architectures," in Radio Frequency Integrated Circuits (RFIC) Symp., Dig. Papers. Jun. 2000

[2] S. Ahson and M. Ilyas, WiMAX: Standards and Security. Boca Raton, Fl: CRC Press, 2008

[3] www.slicklogin.com

[4] http://rnd.azoft.com/mobile-app-transering-data-using-ultrasound

[5] C. Li, D. A. Hutchins, and r. J. Green, "Short-range ultrasonic digital communications in air," IEEE Trans. Ultrasonic. Ferroelectr. Freq. Control, vol. 55, no. 4, pp. 908-918, Apr. 2008.

[6] Chuan Li Hutchins, D.A. Green, R.J., "Short-range ultrasonic communications in air using quadrature modulation",IEEE 2009.

[7] www.embedded.com/Home/PrintView?contentItemId=4024443 